\title{
Performance Analysis of IP Micro-mobility Protocols in Single and Simultaneous Movements Scenario
}

\author{
Giuseppe De $\mathrm{Marco}^{1}$, S. Loreto ${ }^{2}$, and Leonard Barolli ${ }^{1}$ \\ 1 Fukuoka Institute of Technology, \\ Department of Information and Communication Engineering, \\ 3-30-1 Wajiro-Higashi, Higashi-ku, Fukuoka, Japan \\ \{demarco, barolli\}@fit.ac.jp \\ 2 COMICS Lab, Dipartimento di Informatica e Sistemistica, \\ University of Naples "Federico II", via Claudio 21, 80125, Napoli, Italy \\ salvatore.loreto@ieee.org
}

\begin{abstract}
The micro-mobility is an important aspect in ubiquitous communications, where the applications are anywhere and used anytime. In this paper, we analyze two solutions for IP micro-mobility. The first one is based on the Stream Control Transmission Protocol, which allows the dynamic address configuration of an association by means of the ASCONF messages. The second one is based on Session Iinitiation Protocol, which is the most popular protocol for multimedia communication over IP networks. Both in the single and simultaneous movements case, we show that for the SCTP solution, there is space for the further optimization of the handoff latency by using slight changes to the protocol. For the SIP solution, we show that for a correct and fast handoff, the SIP Server should be statefull.
\end{abstract}

\section{Introduction}

Traditionally, the Mobile IP protocol (MIP) has been proposed as network layer (L3) protocol in order to mantain the IP address of mobile node (MN) anywhere it is located [1]. At the application layer, the Session Initiation Protocol (SIP) can also be used to handle MNs 2, 3. If SIP is used as mobility management, all other applications, like Web and FTP, should be modified in order to use the SIP functionalities. Both in SIP and MIP, the network must employ dedicated servers: MIP Home Agents (HA) and Foreign Agents (FA) and/or SIP Server 1 . In order to reduce delays and packet losses due to the handoff process, a lot of variants have been proposed for MIP. An example is the hierarchical MIP, H-MIP and its variants. In general, in all the proposals, the network should be augmented with addtional components, like Mobility Anchor Point in MIP, or

\footnotetext{
${ }^{1}$ SIP Server can be: Registrar Server, Proxy Server, and so on. We use the general word SIP Server by assuming that a dedicated function is inserted in the SIP Server whenever it is required.
} 
MIP Location Registrar (MIP-LR). These proposals are far from the end-toend approach of the current Internet design. In this paper, we compare the SIP based solution against the transport layer based solution to the micro-mobility. As transport layer we use Stream Control Transmission Protocol (SCTP) 4]. To the best knowledge of the authors, this is the first attempt to evaluate if SIP and SCTP can be jointly used for single and simultaneous movement of MNs, without introducing neither additional components in the network nor redundant operations, as we discuss in the following. In Sect. 2, we show different types of architecture of micro-mobility by means of a general taxonomy of mobility functions. In Sect. 3, we analyze the latency caused by handoff process for the case of reliable transmission along with possible optimizations.

\section{Architectures}

To better clarify the IP micro-mobility problems, we use a taxonomy based on the following functions:

- The identitification function, $f: x$-Id $\rightarrow x$-loc, which is used for example when an originating entity (e.g. a correspondent node $\mathrm{CN}$ or another $\mathrm{MN}$ ) wants to communicate with the MN (data are transmitted to or received from it). The originating entity knows the identity of the destination, $x$-Id (e.g. $x$-Id=<giuseppe@sip.fit.ac.jp $>$ ), and needs its locator, $x$-loc.

- The localization and forwarding function, $g: x$-loc $\leftrightarrow$ IP, which maps the $x$ 's locator to a visible IP address and viceversa. The $g$-function is invoked in order to localize the destination IP address, which can vary over time, e.g. whenever an L3 movement takes place in the case of MN travelling among different subnetworks. In general, $g(\cdot)$ is not injective, that is $g$ can have multiple IP addresses associated to its locator.

- The updating function $u:\left(x\right.$-loc, $\left.\mathrm{IP}_{\text {old }}\right) \rightarrow\left(x\right.$-loc, $\left.\mathrm{IP}_{\text {new }}\right)$ wich is usually executed by MN when its IP address has to be changed. The function will

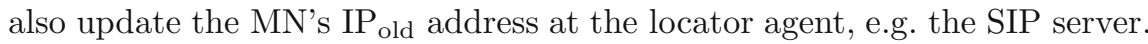

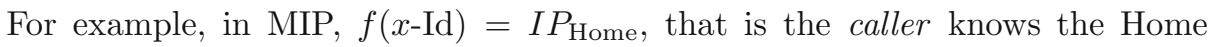
IP address of the destination 2 . Then, the HA performs $g\left(I P_{\text {Home }}\right)=M N_{\mathrm{CoA}}$, that is the HA will forward packets towards the current Care of Address (CoA), $M N_{\mathrm{CoA}}$, of the MN. SCTP can be used for the $u$ function without the support of dedicated location servers as HA and FA in MIP. In fact, SCTP can dinamically add multiple IP addresses during the communication, or association in SCTP terminology, by means of control messages called Association Configuration (ASCONF) 6] [7.

\subsection{MIP+SIP}

In this case, for VoIP applications, the network is redundant, because both SIP Server and MIP-HA perform the same localization function, $f$. This fact has

\footnotetext{
${ }^{2}$ We use the words caller and originating entity as synonymous, although the former
} word is suitable for multimedia communication. For other details see [5]. 


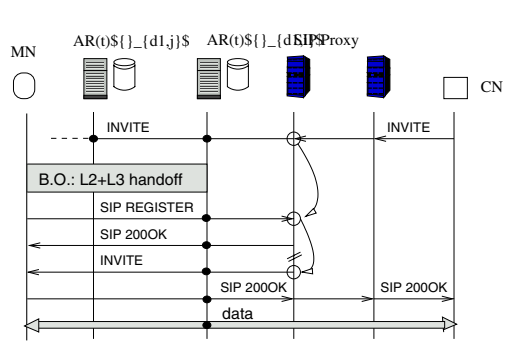

(a) $\mathrm{CN} \longmapsto \mathrm{CN}$ in case of presession mobility

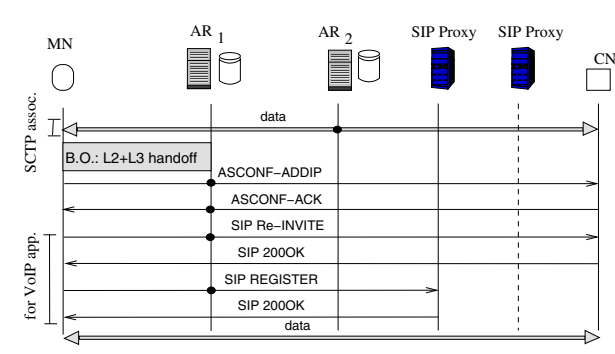

(b) $\mathrm{MN} \mapsto \mathrm{CN}$ in the case of in-session mobility

Fig. 1. Mobility in the case of single movement

been observed also in [8], where the authors proposed to use SIP registration whenever it is necessary. For non-VoIP applications, the function $f$, if required, is executed by standard mechanisms like DNS. Generally to locate the MN, a SIP Server should be employed. Let us note that if route optimization is not available, MIP does also the function $g$ (i.e. the packets forwarding), increasing the overhead 3 , the delays and packet losses, both during the handoff and during the communication. In MIPv6, we can avoids the triangulation problem by means of the direct Return Routability Procedure (RRP) to the CN. Decapsulation is not stricly needed because the CoA address is globally routable. We suppose that the MN can acquire a new CoA by means of a DAD CacheServer, which performs in advance the DAD procedure [9].

\section{$2.2 \quad \mathrm{SCTP}+\mathrm{IPv} 6+\mathrm{SIP}$}

Single Movement. In this architecture, the SIP server is aware of the most updated address of the MN. Whenever the MN enters a new subnetwork, an L2/L3 blackout (BO) takes place, as shown in Fig. 1, and the MN acquires a new CoA by means of IPv6 address auto-configuration. In Fig 1 1 a, the CN initiates the communication (VoIP application) towards the MN by means of the SIP INVITE message. The CN performs the function $f$ by querying its SIP Server (or the SIP server of the MN if both peers belong to the same domain). Since the location stored in the MN's SIP server could be not yet updated, the SIP Server should immediately send a pending INVITE whenever it receives a REGISTER message from the interested MN. In fact, usually the REGISTER message is sent to the SIP Server when the SIP application is made aware of a change of the IP address. The alternative is to wait for timers expiration which will delay the call setup. This could be a serious problem if the MN handoff rate

\footnotetext{
${ }^{3}$ Due to packets decapsulation and triangular forwarding.

${ }^{4}$ This require dedicated support from the kernel sockets.
} 


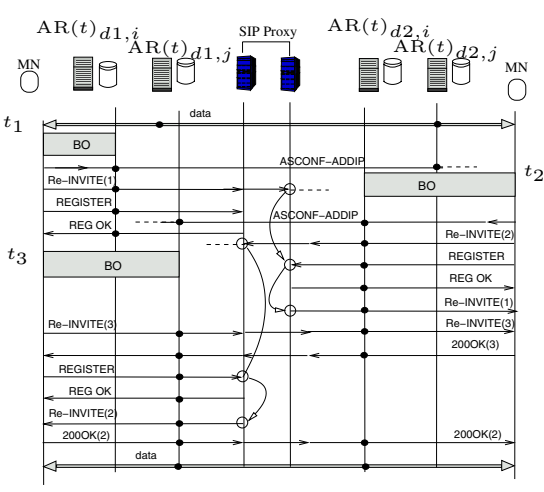

(a) $\mathrm{MN} \mapsto \mathrm{MN}$ with statefull SIP Server

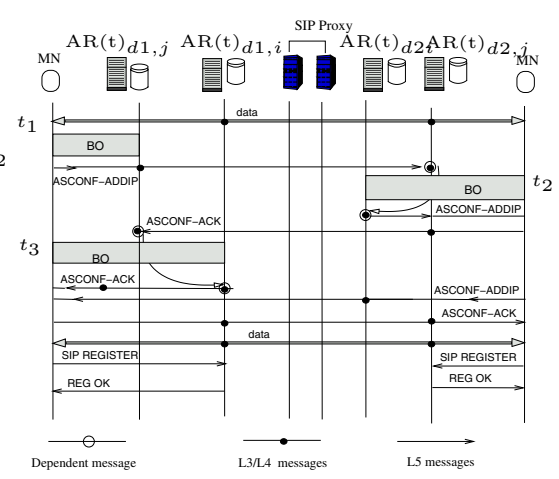

(b) $\mathrm{MN} \mapsto \mathrm{MN}$ with smart $\mathrm{AR}$

Fig. 2. Mobility in the case of simultaneous movements

is high. Accordingly, the SIP Server should be statefull. In both cases, for presession mobility the SCTP does not play a crucial role. On the other hand, if the MN starts non-VoIP communication towards the CN, as in Fig. 1 b the SCTP transport will manage the movements of the MN by means of ASCONF-ADDIP and ASCONF-ACK messages. That is, the SCTP performs the $u$ function inside the MN with $x$-loc $=C N_{\text {IP }}$. No other components are needed.

In this architectures ( $\mathrm{SIP}+\mathrm{SCTP}+\mathrm{IPv} 6)$ redundant registrations are not present. The additional components are: The SIP servers, and the AR supporting IPv6 which are always present in (wireless) subnetwork. One could observe that a full SIP architecture could be enough. But for non-VoIP applications, the function $f, g$ could not be performed because the CN could not have neither a SIP-based domain nor a SIP-compliant application.

Simultaneous Movements. We suppose that the MN's locations are registered at the SIP Server. For example, suppose that at time $t_{1}$ the MN enters a BO phase, and after the L2/L3 handoff, the MN is able to forward and receive packets from $\operatorname{AR}\left(t_{1}\right)_{d 1, i}$, as in Fig. 2 -a-b, that is the $i$ - th $\mathrm{AR}$ visited at time $t_{1}$ and belonging to domain $d 1$. At time $t_{2}$ the $\mathrm{MN}$ in domain 2 moves towards the subnetwork of $\operatorname{AR}\left(t_{2}\right)_{d 2, i}$, and at time $t_{3}$ the MN in domain 1 moves towards $\operatorname{AR}\left(t_{3}\right)_{d 1, j}$. In other words, the MNs can travel along different subnetworks, $i \neq j$, within the same domain, $d 1, d 2$.

\section{Full SIP Solution}

The first solution requires the use of a Re-INVITE message, as in Fig. 27a, because the ASCONF-ADDIP is not able to perform neither the $f$ function nor $u$ function because the ASCONF uses an invalid destination address, that is the IP address of the correspondent peer before its movement. In this case, the use of statefull SIP server is mandatory in order to decrease handoff latency and avoid possible signalling incorrectness, as also pointed out in [10]. 


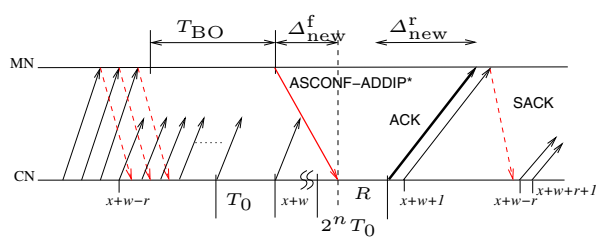

(a) Handoff with timeouts in SCTP standard

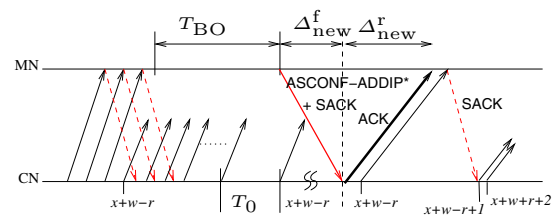

(c)

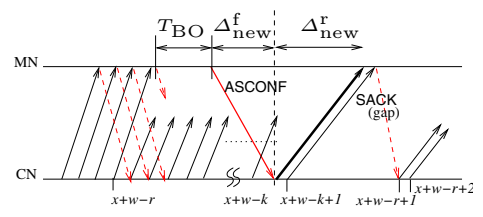

(b) Handoff without timeouts

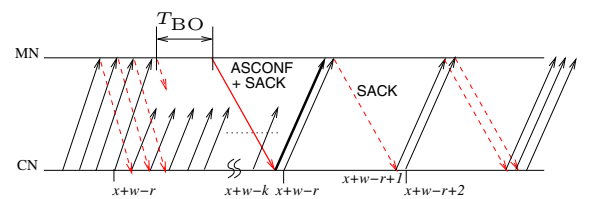

(d)

Fig. 3. Loss recovery for different management of ASCONF-ADDIP: case of standard $\operatorname{SCTP}(\mathrm{a})-(\mathrm{b})$; in (c), the "Version 2" of Algorithm 2 with timeouts, (d) without timeouts

In Fig. 2-b, we show the other solution which does not require the Re-INVITE message and avoid possible breaking of the communication. We suppose that in every domain the $\operatorname{AR}(\cdot)_{d k, i}, k=1,2$, is smart, that is it can store for a certain period of time the new location of the MN just departed. For example, the AR can store an addresses cache for every MN, whose entries being the available addresses for that MN along with their state. Whenever the MN leaves the subnetwork $\operatorname{AR}(\cdot)_{d k, i}$, the cache for that MN will change the state of the old address, e.g. from a "Preferred" state to a "Deprecated" on 5 , and a new "Preferred" address is being added into the cache, i.e. the MN's address acquired in the subnetwork $\mathrm{AR}(\cdot)_{d k, j}$. By this way, the $\mathrm{AR}(\cdot)_{d k, i}$ can forward packets whose destination is "Deprecated" to the proper "Preferred" address, e.g. from $\operatorname{AR}(\cdot)_{d k, i} \operatorname{AR}(\cdot)_{d k, j}$. We do not enter in the detail of the protocol, but only note that this mechanism could be coupled with the DADCache Server discussed previously.

\section{Performance Analysis}

In this section, we analyze the handoff latency for the mobility schemes discussed above. We define latency as the time interval between the last transmission time on the old path and the transmission time of the first new packet on the new path. Firstly, we note that in wired communication the SCTP protocol receives the ASCONF-ADDIP message from the primary path (i.e. the old path in our scenario) and the transmission can advance without interruption. In wireless transmission, the ASCONF message cannot be sent on the primary path because this path is unavailable unless the wireless NIC allows multiple communication

\footnotetext{
5 This mechanism is similar to the addressing scheme of IPv6.
} 


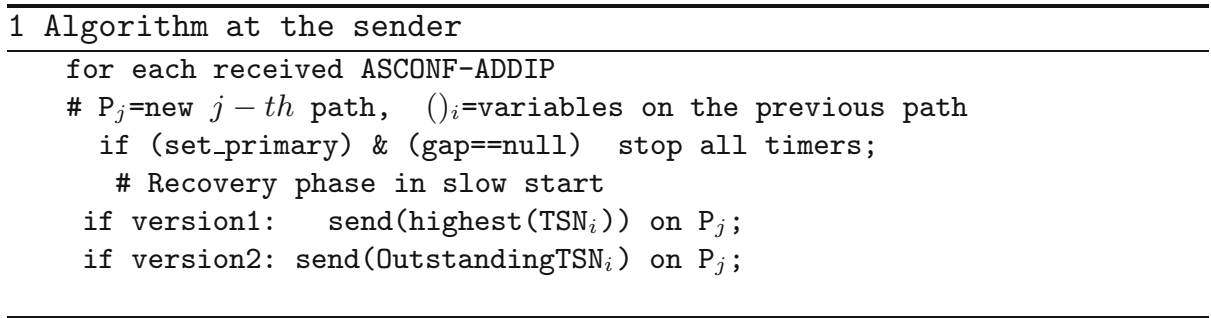

with more than one AP. Accordingly, packet losses and timeouts can occur. Moreover, the transmission over an unknown path should begin with the slow start phase of the congestion control, even if there are enough packets to trigger a Fast Recovery (FR) by sending all the remanining packets over the new path. The solution in [11 respects this contraint, while the authors in [12, without any analysis, suggest to use half of the congestion window on the previous path as the first value of congestion window on the new path. We use slow start phase constraint.

In the case of reliable transmission, the latency is made of two terms: The first one is the delay the sender waits until the arrival of the ASCONF message; the second one is the time of packet loss recovery, as shown in Fig. 3 - a for standard SCTP. If no timeout occur, we have re-transmission according with the congestion control as in Fig. 3.b. We now analyze the latency. For the new path, the transmission delays of the MN-CN and CN-MN path are $\Delta_{\text {new }}^{\mathrm{f}}$ and $\Delta_{\text {new }}^{\mathrm{r}}$, respectively. The $\mathrm{BO}$ phase lasts in $T_{\mathrm{BO}}$ seconds. The number of timeouts during the handover is a random variable, $N$; similarly, the number of lost packets is $L$. For standard SCTP, we note that if the ASCONF-ADDIP message arrives after a timer is started, the protocol sends the next packet after a time $R$ until the expiration of the timer, as in Fig. 3. a. In this case, the mean value of latency for $n$ timeouts is:

$$
E\left\{T_{\mathrm{H}_{n}}\right\}=E\left\{T_{\mathrm{B} 0}\right\}+E\{R\}+E\{L\}+\Delta_{\text {new }}^{\mathrm{f}}+\Delta_{\text {new }}^{\mathrm{r}},
$$

where we suppose $\Delta^{\mathrm{f}, \mathrm{r}}$ being deterministic values, and

$$
\begin{array}{r}
E\{R\}=\sum_{n=1}^{n_{\max }} 2^{n-1} T_{0} P\{N=n\} \\
E\{L\}=\sum_{l=1}^{l_{\max }} R T T\left[\left\lceil\log _{2} l\right\rceil+1\right] P\{L=l\},
\end{array}
$$

where we took into account the slow start phase; $R T T$ is the round-trip time of the new path. In general, for values of the BO phase and the one-way delays under 500ms and for $T_{0}=3 s$, we have $P\{N=1\}=1$ and $P\{N>1\}=0$, that is $E\{R\}=T_{0}$. However, if one accounts also the delays of DAD procedures, the BO phase can be much longer and the probability of more than one timeout increases. The distribution of $L$ in the sum of (3) depends on the congestion 


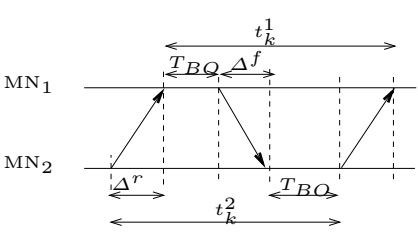

(a)

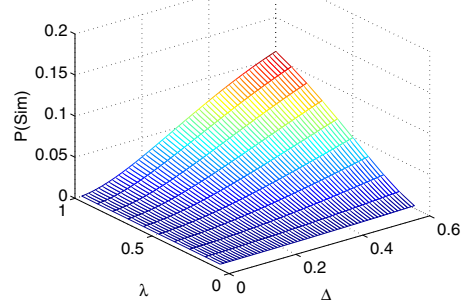

(b)

Fig. 4. (a) Critical times in simultaneous movements and (b) probability $p_{S}$

window size just before the BO phase and the duration of L3 handoff, i.e. until the arrival of ASCONF message. We suppose that the distribution of $L$ is known, although it is difficult to derive a closed form formula. If we use the "version1" of Algorithm 1, we obtain:

$$
E\{L\}=\sum_{l=1}^{l_{\max }} R T T\left[\left\lceil\log _{2} l\right\rceil+1\right] P\{L=l\},
$$

which is the solution of [1]. In that work the packet with $\mathrm{TSN}=x+w+1$ is called probe packet, where $w$ is the congestion window before the handoff. However, in that work is not clear if the ASCONF-ACK is sent or not, what is required by the draft document on ASCONF messages [6]. . In our further optimization, we use the "version2" of Algorithm 2, and then we have:

$$
E\{L\}=\sum_{1}^{l_{\max }} R T T\left[\left\lceil\log _{2} l\right\rceil\right] P\{L=l\},
$$

that is, we can reduce the loss recovery time by one $R T T$. This is possible because the "version2" of the algorithm says that if the ASCONF-ADDIP intends to change the primary path immediatley and if the SACK, bundled in or following the ASCONF, contains a null gap, then the old path is no longer avalaible and the oustanding packets are surely lost. Then, the go-back- $n$ procedure can be promptly activated. This situation is depicted in Fig. 3-d,e. We note that whatever version of the Algorithm 1 we adopt, $E\{R\}=0$, both for $T_{\mathrm{H}_{n}}$ and $T_{\mathrm{H}_{0}}$. In the case of simultaneous movements, the BO phase perceived by each peer can be longer. In the following, we suppose to use Algorithm 1 For simplicity, we focus on the case of Fig. $2 \mathrm{~b}$ and we call $\mathrm{MN}_{1}$ and $\mathrm{MN}_{2}$ the $\mathrm{MN}$ in domain 1 and 2 , respectively; more, we omit the subscript new by supposing that the reverse and the forward path delays are equal for old and new path, respectively. We assume the communication delay between the ARs are negligible and for both MNs the delays of BO phase are identically distributed. Let's name with Sim the event of a deleterious simultaneous movement and with $P(\operatorname{Sim})=p_{S}$ its 
probability. This event happens when both MNs use an erroneous IP address for the destination of packets. After little algebra, (11) writes as:

$$
\begin{array}{r}
E\left\{T_{\mathrm{H}_{n}}\right\}=p_{S} E\left\{T_{\mathrm{H}_{n}} \mid \operatorname{Sim}\right\}+\left(1-p_{S}\right) E\left\{T_{\mathrm{H}_{n}} \mid \overline{\operatorname{Sim}}\right\}= \\
=p_{S} E\{L \mid \operatorname{Sim}\}+\left(1-p_{S}\right) E\left\{L \mid \overline{\operatorname{Sim}\}}+\Delta^{\mathrm{f}}+\Delta^{\mathrm{r}}+\left(1+p_{S}\right) E\left\{T_{\mathrm{BO}}\right\}\right.
\end{array}
$$

The probability of Sim depends on the interval the MNs spend to change the IP address and the rate of the handoff, i.e. $\lambda_{1}=\frac{1}{E\left\{t^{1}\right\}}$ and $\lambda_{2}=\frac{1}{E\left\{t^{2}\right\}}$. From Fig. $4 \mathrm{a}$, it is easy to recognize that the critical interval during which a simultaneous movement can happen is $\Delta^{\mathrm{r}}+\Delta^{\mathrm{f}}$. In fact, the ADDIP of $\mathrm{MN}_{2}$ arrives at $\mathrm{MN}_{1}$ in after a time of $\Delta^{\mathrm{r}}$ and similarly for the ADDIP of $\mathrm{MN}_{1}$. Thus, the probabiliy of $\mathrm{Sim}$ is the probability of an handoff arrival in these intervals. If we suppose that the handoff interarrival times are exponentially distributed with means $\lambda_{1}$ and $\lambda_{2}$ for $\mathrm{MN}_{1}$ and $\mathrm{MN}_{2}$, respectively, we have:

$$
p_{S}=e^{-\lambda_{1} \Delta^{\mathrm{f}}} e^{-\lambda_{2} \Delta^{\mathrm{r}}} \lambda_{1} \lambda_{2} \Delta^{\mathrm{f}} \Delta^{\mathrm{r}} .
$$

In Fig. 4 b, we plot an example of this probability, for $\Delta^{\mathrm{f}, \mathrm{r}}=\Delta$ and $\lambda=\lambda_{1,2}$. As shown, the probability of simultaneous movements is not negligible as the handoff rate and the path delays increase [10. In general, the handoff rate depends on the size of the radio cell and the velocity of the MN and can be high especially in ubiquitous applications like inter-vehicle communications. We observe that $p_{S}$ is also the probability of communication drop if the smart ARs are not employed. In the simultaneous movements case, $E\{L \mid S i m\}$ is more complicated, because the number of lost packets depends also on the direction of the communication.

For single movements and SIP scenario, as in Fig. 1 b b, the latency is as in (1), because the the SIP Re-INVITE and SIP 200OK messages are the equivalent of ASCONF-ADDIP and its ACK. The slight difference would be that the socket needs not to be re-opened. For simultaneous movements, the latency can be longer. In fact, as shown in Fig. 2 a, the $\mathrm{MN}_{2}$ must wait the arrival of the SIP Re-INVITE message in order to "re-open" the connection with the new IP of $\mathrm{MN}_{1}$. This message is sent by SIP Server of $\mathrm{MN}_{2}$, after the reception of the REGISTER message: If the SIP proxy is far, the latency will be higher than that of the SCTP case. In other words, the affected parameters are $\Delta^{\mathrm{r}}$ and $\Delta^{\mathrm{f}}$. Accordingly, the latency would be:

$$
E\left\{T_{\mathrm{H}_{n}}\right\}_{\mathrm{SIP}} \geq E\left\{T_{\mathrm{H}_{n}}\right\}_{\mathrm{SCTP}},
$$

with obvious meaning of the subscript.

\section{Discussion}

In this paper, we recognized that some combinations of mobility protocols, like $\mathrm{MIP}+\mathrm{SIP}$, introduce redundancy in the network. With a kind of criticism, we saw that if the standard applications, like Web and FTP, are not supported by SIP based domains, the micro-mobility can not be realized with SIP. On 
the other hand, transport layer solutions to the micro-mobility problems are possible whenever the protocol permits the dynamic change of the destination IP address, like SCTP with ASCONF messages. This solution does not require SIP domains. In Sect. 2] we analysed a possible optimization of the handoff latency when ASCONF is used: If the ASCONF contains a "set-primary" parameter, the timers should be stopped, otherwise there is a mean term of $T_{0}$ (usually set to $3 \mathrm{~s}$ ) in the latency, at least when the congestion window is less than 2 packets. In Sect. 3, we recognized the following results about simultaneous mobility.

- The optimization of ASCONF is much more important, because $T_{B 0}$ can be much longer and then $E\{R\}$ could be greater than $T_{0}$.

- The use of SCTP requires changes into the ARs (smart AR).

- The use of SIP requires statefull SIP Server

- The more the SIP Servers are far from the subnetwork which the MN is moving towards, the more the use of SIP increases the latency of the handoff. We do not illustrate all possible cases because of page constraints.

As a matter for further investigation, we note that subnetworks equipped with the smart AR could solve both problems of SCTP, for the updating function, and SIP, for the decrease of the handoff latency.

\section{References}

1. D.Johnson, C.Perkins, J.Arkko: Mobility Support in IPv6. IETF RFC 3775. (2004)

2. : (IETF Sip Working Group)

3. Nakajima, N., Dutta, A., S.Das, Schulzrinne, H.: Handoff delay analysis and measurement for sip based mobility in ipv6. In: Proceedings of ICC'2003. Volume 26. (2003) 1085-1089

4. R.Stewart, et al.: Stream Control Transmission Protocol. (IETF RFC 2960)

5. Marco, G.D., Loreto, S., L.Barolli: Performance analysis of ip micro-mobility protocols in single and simultaneous movements scenario. Technical report, FIT (2005)

6. Stewart, R., other: Stream Control Transmission Protocol (SCTP) Dynamic Address Reconfiguration. IETF - draft. (2004)

7. Xing, W., Karl, H., Wolisz, A., Müller, H.: M-sctp: Design and implementation of an end-to-end mobility concept. In: Proceeding of 5th Workshop The Internet Challenge: Technology and Applications. (2002)

8. H.Lee, S.W.Lee, Cho, D.H.: Mobility management based on the integration of mobile ip and session initiation protocol in next generation mobile data networks. In: Proceedings of IEEE Vehicular Technology Conference. Volume 3. (2003)

9. Hwang, S.H., Y.H.Han, Hwang, C.S., Min, S.G.: An address configuration and confirmation scheme for seamless mobility support in ipv6 network. In: Proceedings of Wired/Wireless Internet Communications Conference. Volume 2957 of LCNS., Springer-Verlag (2004) 74-86

10. Wong, K., Dutta, A., K.Young, H.Shulzrinne: Managing simultaneous mobility of ip hosts. In: Proceedings of MILCOM'03. Volume 22. (2003) 785-790

11. Chang, M., M. Lee, H.L.Y.H., Park, J.: An enhancement of transport layer approach to mobility support. In: Proceeding of ICOIN'05. LCNS, Springer-Verlag (2005) 864

12. Aydin, I., Seok, W., Shen, C.C.: Cellular sctp: a transport layer approach to internet mobility. (In: Proceedings of IEEE ICCCN'03) 285-290 